\title{
Influence of intrinsic kink-like defects on screw dislocation - coherent twin boundary interactions in copper
}

\author{
Qiongjiali Fang ${ }^{\mathrm{a}}$ and Frederic Sansoz, ${ }^{\mathrm{a}, \mathrm{,}}$
}




\begin{abstract}
The interaction mechanisms between a dislocation and a twin boundary in nanotwinned face-centered cubic metals are well understood in terms of perfect coherent interfaces. Processes involving intrinsic incoherent twin boundary defects, however, remain largely unexplored, despite recent evidence suggesting that imperfect twin boundaries containing short kink-like step defects contribute notably to plastic deformation and twin stability in large nanotwinned grains. Here, molecular dynamics simulation is used to study the underlying interaction of screw dislocations with either $0^{\circ}$ or $60^{\circ}$ atomic-scale kink twin boundary defects, in order to understand how the presence of imperfect twin boundaries can alter hardening and ductility mechanisms in nanotwinned copper. It is found that kinked twin boundaries are effective in changing the mechanisms from direct dislocation transmission to dislocation absorption when the applied shear strain exceeds $1.06 \%$, with pronounced hardening arising from such transformation. Hardening by dislocation pinning from individual kink steps is also manifest for $60^{\circ}$ intersections. On the contrary, twin boundary defects produce no hardening at low applied strains when dislocation absorption at the twin boundary is already prevailing.
\end{abstract}

Keywords: Dislocation; Coherent twin boundary; Incoherent twin boundary; Molecular dynamics simulations 


\section{Introduction}

Face-centered cubic (fcc) metals with ultrafine grains ( $>100 \mathrm{~nm}$ ) containing nanoscale coherent twin boundaries (CTBs) formed by crystal growth [1,2], plastic deformation or recrystallization $[3,4]$ have proven to exhibit superior tensile strength and ductility than nanocrystalline fcc metals (grain size $<100 \mathrm{~nm}$ ). Also the low excess energy of CTBs has shown to increase twin stability in high temperature and radiation environments [5-7], as well as thermal conductance, electrical conductivity and corrosion resistance [8-10], compared to general grain boundaries. The mechanical behavior of nanotwinned metals is strongly influenced by two microstructure dimensions, the mean CTB spacing $(\lambda)$ and the average grain size $(d)$. Nanotwinned $\mathrm{Cu}$ metals containing equiaxed-polycrystalline grains with $d \sim 500 \mathrm{~nm}$ and $\lambda \sim 15 \mathrm{~nm}$ have been found to achieve optimum strengths near $1 \mathrm{GPa}$ with impressive uniform elongations before failure up to $14 \%$ strain $[9,11]$. The simultaneous rise in strength and ductility is generally governed by work hardening effects from dislocation-CTB interactions that increase dramatically when $\lambda$ decreases to the nanoscale [12-14]. Furthermore, dislocation-CTB interaction mechanisms have been found responsible for significant plastic anisotropy in columnar-grained $\{111\}$ textured nanotwinned fcc metals, in which crystal slip becomes more sensitive to the grain orientation with respect to loading direction [15-22], because stress concentrations from grain boundary triple junctions are minimized [23-25].

Fig. 1(a) presents a Thompson tetrahedron depicting all possible $\{111\}$ slip planes (ABD, ACD, BCD) intersecting a $53(111) \mathrm{CTB}(\mathrm{ABC})$ in an fcc metal. The 
associated slip modes can be divided into three categories [16]. First, glide of a partial dislocation (e.g. $\mathrm{A} \delta, \mathrm{B} \delta$ or $\mathrm{C} \delta$ ) on the $\mathrm{CTB}$ plane is a soft mode because the dislocation moves easily over a large domain $d$, offering significant space between grain boundaries for multiplication of twinning partial dislocations. Second, a dislocation can glide on a slip plane intersecting the CTB (e.g. ABD) with a slip vector parallel to the twin plane (e.g. AB). This slip mode is hard due to the collective motion of threading dislocations confined within a single twin layer [19] or necklacelike extended jogged dislocations [26]. Third, a dislocation can glide on a slip plane intersecting the CTB (e.g. ABD) with a non-parallel slip vector (e.g. DA or DB). This mode is expected to produce maximum hardening due to the limited space for dislocation glide within two CTBs, and the large elastic energy barrier from the slip discontinuity across CTBs [27].

Accurate prediction of twin boundary (TB)-induced strengthening in different fcc metals, however, requires detailed atomistic understanding on dislocation-twin interactions [28]. Specifically, recent molecular dynamics (MD) simulation studies [29-31] have focused on interaction mechanisms between an implanted screw dislocation and a CTB inside bicrystals. It has been established that a screw dislocation constricts to a full dislocation at the intersection with a CTB and cross-slip via the Friedel-Escaig mechanism, either forming two partial dislocations gliding in opposite directions on the twin plane, or transferring through the CTB to form a new full dislocation in the adjacent twin grain. Atomistic simulations have shown that both constriction behavior and dislocation pathways are strongly material-dependent. Jin et al. [30,32] simulated the motion of a screw dislocation forced toward a CTB by 
dynamic relaxation at a constant applied strain. In $\mathrm{Cu}$ and $\mathrm{Ni}$, these authors have found that the leading partial only enters the CTB when the shear strain is high enough to overcome the repulsive force from the CTB [27]. In contrast, for Al, a screw dislocation will spontaneously combine into a full dislocation at the CTB, because the interaction force between screw dislocation and CTB becomes attractive in this metal below a standoff distance of $4 \mathrm{~nm}$. Furthermore, after constriction, a screw dislocation in $\mathrm{Cu}$ or $\mathrm{Ni}$ dissociates into two twinning partial dislocations moving in opposite directions under low shear strain. This process will be referred to as mechanism A (absorption) in the following. Using notations shown in Fig. 1(b), this reaction can be written as

$$
A \gamma+\gamma B \rightarrow A B \rightarrow A \delta+\delta B
$$

Under higher shear strain, the same full dislocation directly transmits through the CTB and is re-dissociated into two partial dislocations on a different $\{111\}$ slip plane in the twin grain, which will be referred to as mechanism $\mathrm{T}$ (transmission), such as

$$
A \gamma+\gamma B \rightarrow A B\left(B^{\prime} A^{\prime}\right) \rightarrow B^{\prime} \gamma^{\prime}+\gamma^{\prime} A^{\prime}
$$

For Al, however, Jin et al. have only observed mechanism A under all applied shear strains. Moreover, past studies have reported other types of dislocation interaction with CTBs; for example, the Fleischer mechanism involving two partials of a dissociated screw dislocation directly transmitted across the CTB without constriction $[33,34]$, or the formation of sessile stair-rod and Lomer dislocations with non-screw dislocations or mixed dislocation loops [12,32-35]. Also, Ni et al. [36] found that the 
dislocation density could have an influence on dislocation-twin interaction mechanisms.

It is important to emphasize that CTBs are not always perfect. Short segments of incoherent $\Sigma 3\{112\}$ TBs (ITBs) are routinely observed in electrodeposited and sputtered nanotwinned $\mathrm{Cu}$ and $\mathrm{Ag}$ metals by transmission electron microscopy (TEM) $[2,37]$. Even if ITBs are not detectable by standard TEM, a recent study by Wang et al. [38] using sub-nm-scale inverse pole figure orientation mapping on magnetronsputtered nanotwinned $\mathrm{Cu}$, has identified the existence of intrinsic kink-like defects on CTBs of $\sim 1-5 \mathrm{~nm}$ in height. These defects were significantly larger than the height of twinning partial dislocation loops $(0.147 \mathrm{~nm})$ previously reported on CTBs $[11,24]$, but also typically shorter than the ITBs observed in epitaxial nanotwinned $C u$ films or those formed by twinning/detwinning of fcc metals during deformation [3942]. The formation mechanism of these short kink-like steps along otherwise straight CTBs, however, currently remains an unresolved question, but is expected to be linked to processing parameters. In-situ synchrotron X-ray diffraction experiments and MD simulations [38] have shown that kink TB defects in these imperfect CTBs play important roles on plastic deformation and twin stability in nanotwinned $\mathrm{Cu}$. TB defects can migrate under very low applied shear strain or become pinning points to stop the motion of threading dislocations. In addition, kink TB defects influence stress concentrations responsible for the nucleation of new dislocations [25]. Yet, our current understanding of dislocation processes involving defective CTBs is still limited. 
In this article, MD simulations are used to study the influence of intrinsic kink-like TB defects on interaction mechanisms between a screw dislocation and a CTB in $\mathrm{Cu}$ bicrystals, similar to those used in past atomistic simulation studies for perfect CTBs $[30,31]$. The specific objectives of this study are (1) to understand how absorption and transmission mechanisms for screw dislocations intersecting CTBs are altered in the presence of a kink TB defect, and (2) to examine how twin-induced hardening effects change from perfect to imperfect CTBs. Section 2 presents the atomistic simulation methodology for modeling interaction mechanisms in nanotwinned $\mathrm{Cu}$ bicrystals. Atomic-scale microstructure and deformation mechanisms between a screw dislocation and a defective CTB containing either $0^{\circ}$ or $60^{\circ}$ oriented kink defects subjected to different constant shear strains, are presented in Section 3. The roles of kink TB defects on dislocation absorption and transmission mechanisms, and how they relate to strengthening and ductility in nanotwinned $\mathrm{Cu}$, are discussed in Section 4.

\section{Methodology}

MD simulations were performed using the software LAMMPS [43] with the embedded-atom-method potential for $\mathrm{Cu}$ developed by Mishin et al. [44]. This potential has shown to provide a good match with ab-initio calculations of stackingfault and twin-formation energies in this metal [45]. A Cu bicrystal containing two parallel CTBs was modeled as schematically shown in Fig. 1(c). Crystal orientations in the matrix and twin grains were $x$-[112], $y$-[ $[\overline{1} 10], z-[\overline{1} \overline{1} 1]$ and $x-[552], y-[1 \overline{1} 0]$, $z$ - $[11 \overline{5}]$, respectively. The dimensions of the simulation box were $L_{x}=431.1 \AA, L_{y}=$ 
$15.4 \AA$ and $L_{z}=250.5 \AA$. Periodic boundary conditions were applied in the $x$ and $y$ directions to minimize surface effects. We also found that simulation with a model 20 times larger in the $y$ direction achieved the same results. The energy of the model was first minimized at $0 \mathrm{~K}$ by conjugate gradient method. MD simulations were then conducted under microcanonical ensemble (NVE) with a timestep of 2 fs. The initial temperature was set to be $0.1 \mathrm{~K}$ in order to limit thermal activation of dislocation processes.

A single screw dislocation of Burgers vector $b_{s}=a_{0} / 2[\overline{1} 10]$ with $a_{0}$ the lattice parameter, was added on the left-hand side of the model by rigidly moving two groups of 6 atom layers, as shown by magenta and blue-colored regions in Fig. 1(c), by the same distance in the $x y$ plane, but in opposite directions, while keeping all other atoms relaxed. The first displacement of the magenta group was $a_{0} \sqrt{6} / 12$ in the [121] direction, and the second one was $a_{0} \sqrt{6} / 12$ in the [ $\left.\overline{2} 1 \overline{1}\right]$ direction, each displacement applied over 5,000 MD steps. The resulting screw dislocation was dissociated into two partials with a stacking fault ribbon of $\sim 11 \AA$ in width.

A constant shear strain parallel to the $y z$ plane was imposed to the model by applying a displacement increasing linearly from bottom to top, Fig. 1(d). Three atom layers at the top and bottom, Fig. 1(c) and (d), were fixed in subsequent MD steps, while all other atoms were allowed to relax. The overall von-Mises stress was computed during dynamic relaxation by using the virial theorem and the deformed volume. Different shear strains varying between $0.8 \%$ and $4.0 \%$ were simulated. Microstructures and deformation mechanisms were studied at atomic scale with the adaptive common 
neighbor analysis (CNA) $[46,47]$ and dislocation extraction analysis (DXA) $[48,49]$ in the atomistic visualization software OVITO [50].

Kink-like step defects were introduced in one CTB as shown in Fig. 2. The defect line was perpendicular to one of the $<112>$ directions in the CTB, at either $0^{\circ}$ or $60^{\circ}$ angle with respect to the screw dislocation line, Figs. 2(a) and 2(d), respectively. For $0^{\circ}$ kink steps, a single defect was added at two different positions on the CTB, either at a distance $x=37 \AA$ above the slip plane of the dislocation or immediately at the plane intersection with the CTB ( $x=0 \AA$ ), Fig. 2(b). For $60^{\circ}$ kink steps, Fig. 2(e), a full kink defect made of two steps was added in such a way as to maintain periodic boundary conditions along the $y$ direction, which was increased to $20 \times L_{y}$ for that purpose. The distance between each kink step along the $y$ direction was equal to 123 $\AA$.

The structure of kink TB defects modeled in this study consisted of a short $\Sigma 3\{112\}$ segments made by stacking three $\{111\}<112>$ dislocations in a repeatable $b_{2}: b_{1}: b_{3}$ pattern [41], as shown in Fig. 2(c). Here $b_{1}=C \delta=1 / 6[\overline{1} \overline{1} 2]$ is a pure edge partial dislocation, $b_{2}=A \delta=1 / 6[\overline{1} 2 \overline{1}]$ and $b_{3}=B \delta=1 / 6[2 \overline{1} \overline{1}]$ are mixed partial dislocations [40], and the sum of the three Burgers vectors is null [51].

\section{Results}

\subsection{Dislocation absorption and transmission mechanisms with perfect CTBs}

Fig. 3 presents dislocation processes involving only perfect CTBs under two applied shear strains, $0.8 \%$ and $1.2 \%$, which confirmed the two interaction mechanisms 
reported in the literature [30,31]. Fig. 3(a) shows that cross-slip by dislocation absorption at the CTB (mechanism A) takes place at a strain of $0.8 \%$. The screw dislocation moves toward the $\mathrm{CTB}$, and becomes constricted into a full dislocation at the slip-CTB intersection after 6.8 ps, followed by absorption and re-dissociation into two partials on the CTB plane. This reaction is found to satisfy Eq. (1). At shear strain of $1.2 \%$, the constriction behavior at the CTB is similar, but Fig. 3(b) shows that the constricted dislocation is directly transferred to the twin grain (mechanism T) where it becomes a new dissociated screw dislocation gliding on a different $\{111\}$ plane.

By conducting several atomic simulations at different strains between $0.8 \%$ and $1.2 \%$, we determined that the transition between mechanisms $\mathrm{A}$ and $\mathrm{T}$ is close to $1.06 \%$ strain. It is worth noting that the temperature increased sharply within 1 ps after relaxation, but also did not exceed $2.5 \mathrm{~K}$ during the simulation. We studied the effect of temperature by adding a Berendsen thermostat in the simulations, in order to maintain a constant temperature near $0.1 \mathrm{~K}$. It was found that the threshold transition strain from absorption to transmission mechanism in perfect CTBs increased from $1.06 \%$ to $1.28 \%$ using the thermostat.

\subsection{Interaction mechanisms with $0^{\circ}$ kink $T B$ defects}

First, we address the situation where a $0^{\circ}$ kink step is present on the $\mathrm{CTB}$, but is not directly aligned with the slip plane of the screw dislocation $(x=37 \AA)$. Fig. 4 shows that the mechanisms in this case remain identical to those for perfect CTBs, i.e. the occurrence of mechanisms $\mathrm{A}$ and $\mathrm{T}$ at low and high shear strains, respectively. It was also noticed that at high strains, the TB step could migrate along the CTB, as shown in Fig. 4(b). Self-migration of the kink steps without dislocation interaction will be 
discussed below in Section 4.4. The step migration, however, was found to be inconsequential for the dynamic stress relaxation presented in Section 3.4.

Second, when the dislocation directly intersects a $0^{\circ}$ kink step defect $(x=0 \AA)$, our MD simulation results in Fig. 5 predict several changes in dislocation dynamics at the TB-slip intersection. At low strain of $0.8 \%$, Fig. 5(a), the dislocation combines with the kink TB step at $6 \mathrm{ps}$, and then dissociates into three twinning partials; one moves upwards and the other two move downwards along the CTB. Since this mechanism relates to CTB absorption with the involvement of three partials instead of two, we call this mechanism $\mathrm{A}^{\prime}$ by distinction with mechanism A for perfect CTBs. At intermediate shear strain of $1.2 \%$, Fig. $5(\mathrm{~b})$, the dislocation transmission initially predicted with perfect CTBs changes dramatically from mechanism $\mathrm{T}$ to absorption mechanism A'. Furthermore, when the shear strain is increased up to $2 \%$, Fig. 5(c), a new absorption mechanism arises, mechanism A", involving three partial dislocations moving in the same direction on the CTB, which differs from mechanism A'.

\subsection{Interaction mechanisms with $60^{\circ}$ kink TB defects}

Movies S1-S3 present the simulations of a screw dislocation intersecting a $60^{\circ}$ kinked CTB under different applied strains. The dislocation is found to react with each of the two kink steps present, thereby dividing the dislocation line into three segments. Under $0.8 \%$ shear strain, Fig. 6 and Movie S1 show that each dislocation segment is predicted to dissociate by cross-slip on the twin plane via mechanism A and no transmission to the twin grain. It is important to note, however, that twinning partials moving on the CTB are directly formed by the kink defects, suggesting that these 
partials are a direct product of an energetically favored reaction between the incoming dislocation and the TB defects.

When the shear strain is increased to $1.2 \%$, Fig. 7 and Movie S2, we observe a mixed mode where the middle segment of the dislocation dissociates on the TB by mechanism A, while the front and back segments transmit to the twin grain by mechanism $\mathrm{T}$, and the ends of the transmitted dislocations are pinned by kink steps. As relaxation continues, the middle segment evolves to full transmission, and forms a single dissociated screw dislocation together with the formerly transmitted segments in the front and back. We studied the complex dislocation process in the middle segment by DXA [48,49], and the results of this analysis are represented schematically in the right column of Fig. 7 and Movie S4. First, the screw dislocation dissociates into two partials on the CTB following the reaction described by Eq. (1). During relaxation, one twinning partial $A \delta$ becomes a leading partial in the twin grain and a stair-rod dislocation on the CTB such as

$$
A \delta \rightarrow A \gamma^{\prime}+\gamma^{\prime} \delta
$$

Subsequently, the second twinning partial combines with the newly formed stair-rod dislocation, and turns into a new trailing partial in the twin grain, according to the reaction

$$
\gamma^{\prime} \delta+\delta B \rightarrow \gamma^{\prime} B
$$

At high strain of $2.0 \%$, Fig. 8 and Movie S3 show that mechanism $\mathrm{T}$ is predicted in all segments. However, the interaction of the middle segment with the TB leads to the transmission of a dislocation into the twin grain on a slip plane that is parallel to that 
of the left and right segments, but also separated by two atom planes, which forms a jogged dislocation. In addition, the dislocation is firmly pinned to the kink steps after transmission.

\subsection{Dynamic stress relaxation}

The role of kink TB defects on dynamic stress relaxation under constant strain was investigated by comparing the difference in von-Mises stress between the models, as a function of simulation time. In absence of CTBs, our simulations predict a linear stress decrease during propagation of the screw dislocation in a single crystal subjected to a constant shear strain, as shown by dashed lines in Fig. 9. When perfect or imperfect CTBs are added to the model, a change in dynamic relaxation occurs at the intersection of the dislocation with the first CTB, which is represented by a black arrow in Fig. 9. Here, hardening behavior is signaled by a rise in the curve slope when the CTB impedes dislocation movement.

For interactions with $0^{\circ}$ kink defects under low shear strain of $0.8 \%$, Fig. 9 suggests no significant variations in stress when only absorption mechanisms are involved, although mechanisms $\mathrm{A}, \mathrm{A}^{\prime}$ and $\mathrm{A}^{\prime}$ are slightly different. It can therefore be concluded that the presence of kink defects plays no role in hardening or softening the metal at low applied strain, compared to perfect CTBs. At shear strains above $1.2 \%$ under which direct dislocation transmission is expected with perfect CTBs, however, Fig. 9 reveals significant hardening effect in the dynamic response for defective TBs, more so when the shear strain is raised up to $2 \%$. This phenomenon can be attributed to the transformation from transmission mechanism $\mathrm{T}$ to absorption mechanisms $\mathrm{A}^{\text {' }}$ or A". Furthermore, Wang et al. [40] proposed another possible structure for the core 
of the kink step involving 4 partial dislocations instead of 3. The stress-time behavior from this other defect structure was also simulated in this study. Same hardening was found when the mechanism changed from transmission to absorption due to kink defects.

For interactions with $60^{\circ}$ kinked TBs, Fig. 9 shows that hardening is also manifest, but to a lesser extend than for $0^{\circ}$ kink defects. The highest hardening effect is predicted for intermediate strains of $1.2 \%$ where the middle segment is forced to cross-slip along the CTB via Eq. (3)-(4), instead of directly transmitting into the twin grain. Under high strain of $2 \%$, the transmitted dislocation is divided into three segments with two pinning points at each kink step. Dynamic stress relaxation reveals moderate hardening by dislocation pinning, compared to complete transmission in perfect CTBs.

\section{Discussion}

\subsection{Strain dependence of screw dislocation-CTB interaction mechanisms}

To understand the transition from absorption to direct transmission in different fcc metals, Jin et al. [30] have first developed a material-dependent and energy-related criterion (minimum energy barrier) based on the competition between partial dislocation nucleation in the twin grain (mechanism $\mathrm{T}$ ) and nucleation of twinning partials on the CTB (mechanism A). Their predictions led to a threshold strain in the $1.0-1.1 \%$ range corresponding to stresses between $465 \mathrm{MPa}$ and $510 \mathrm{MPa}$ in $\mathrm{Cu}$, but no apparent threshold in Al. Chassagne et al. [31], however, argued that this criterion was not accurate, and proposed that the mechanism transition from absorption to 
transmission takes place at the same stress among different fcc metals, which corresponds to that required for the dislocation constriction at the CTB-slip intersection. They established that direct transmission occurs when the constriction stress is larger than $400 \mathrm{MPa}$, except for $\mathrm{Al}$ where screw dislocations are spontaneously absorbed by the interface. In the present study, given that the $\{111\}<110>$ shear modulus is $41 \mathrm{GPa}$ with the potential by Mishin et al. [40], the threshold shear strain between mechanism A and mechanism T, i.e. $1.06 \%-1.28 \%$ at $0.1 \mathrm{~K}-2.5 \mathrm{~K}$, corresponds to shear stresses between $435 \mathrm{MPa}$ and $525 \mathrm{MPa}$. This value agrees well with Chassagne et al.'s and Jin et al.'s results in $\mathrm{Cu}$ bicrystals, although Jin et al. refitted Mishin's potential to $0 \mathrm{~K}$ properties, and did not impose periodic boundary conditions along the $x$ direction.

When $0^{\circ}$ kink TB defects were introduced, but the dislocation did not directly interact with a kink step, our simulations in Fig. 4 have shown no change in interaction mechanisms. However, simulating the intersection of a screw dislocation with this type of TB defects $(x=0 \AA)$ showed no clear strain-dependence on dislocation-twin interactions, because transmission mechanism $\mathrm{T}$ was systematically change into absorption mechanisms A' or A", therefore eliminating the threshold strain altogether. Adding $60^{\circ}$ kink TB defects produced mixed deformation modes with a gradual change from mechanism $\mathrm{A}$ to $\mathrm{A} / \mathrm{T}$ and ultimately mechanism $\mathrm{T}$ with pinning, as the applied shear strain was increased. It was found that dislocation absorption by the CTB was possible up to $1.12 \%$ strain without imposed thermostat, but also this limit increased up to $\sim 2 \%$ with a Berendsen thermostat, which is similar to the temperature effect observed in perfect CTBs discussed in Section 3.1. Apparently, in $60^{\circ}$ kink 
models, the average dislocation speed before constriction was found to decrease from $\sim 1500 \mathrm{~m} \cdot \mathrm{s}^{-1}$ to $\sim 977 \mathrm{~m} \cdot \mathrm{s}^{-1}$ under shear strain of $2 \%$ when switching the thermostat. Therefore, this observation could suggest that the increased threshold strain with thermostat results from a decrease in dislocation speed that affects the stress relaxation over time.

\subsection{Influence of $0^{\circ}$ kink $T B$ defects on interaction and hardening mechanisms}

When the screw dislocation comes in direct contact with a $0^{\circ}$ kink step, a major finding of this study is that the dislocation is always absorbed into the CTB regardless of the applied shear strain. The reaction leads to either mechanism A' or A", involving propagation of three twinning partials from the kink step. It is possible to understand the new dislocation product by considering the reaction between the screw dislocation and one of the two mixed partials, for example,

$$
A \gamma+\gamma B+b_{3} \rightarrow A B+B \delta \rightarrow A \delta
$$

or equivalently,

$$
\left.A \gamma+\gamma B+\text { kinkTBdefect } b_{3}: b_{1}: b_{2}\right) \rightarrow A \delta+b_{1}+b_{2}
$$

Before interaction, there are five dislocations: Two partial dislocations from the dissociated screw dislocation, $A \gamma=\frac{1}{6}[\overline{2} 1 \overline{1}]$ and $\gamma B=\frac{1}{6}[\overline{1} 21]$, and three partial dislocations from the kink TB step, $b_{1}+b_{2}+b_{3}$, whose Burgers vectors add up to zero. As mentioned in Section 2, $b_{1}=C \delta=1 / 6[\overline{1} \overline{1} 2], b_{2}=A \delta=1 / 6[\overline{1} 2 \overline{1}]$ and $b_{3}=B \delta=1 / 6[2 \overline{1} \overline{1}]$. After interaction, there are three dislocations: $A \delta+b_{1}+b_{2}$ with a total Burgers vector equal to $\frac{1}{2}[\overline{1} 10]$. Therefore, the Burgers vector of the initial screw dislocation is conserved before and after interaction. 
Eq. (6) shows that this type of interaction produces two mixed partial dislocations moving in opposite directions (A $\delta$ and $b_{2}$ ) and one edge partial dislocation $\left(b_{1}\right)$. As shown in Fig. 5, the edge dislocation is attracted by one of the two twinning partials to form a dislocation dipole. To summarize, if the dislocation hits the top of a $0^{\circ}$ kink $(x=0 \AA)$, interaction with $b_{3}$ occurs and mechanism A' is observed. But, if the slip plane intersects the other mixed dislocation $b_{2}$, i.e. if the dislocation hits the bottom of a $0^{\circ}$ kink $(x=2 \AA)$, the twinning partial is predicted to migrate in the opposite direction, and consequently mechanism A" is observed. Interestingly, mechanism A" was also predicted in Fig. 5(c) between a screw dislocation and a $0^{\circ}$ kinked CTB at $x$ $=0 \AA$ under high shear strain of $2 \%$. By comparison, we found that a common feature in both situations is the structure of the constricted dislocation at the twin-slip intersection. Therefore a new hypothesis from this study is that the displacement of twinning partials migrating either upwards or downwards along the CTB with mechanisms A' and A", relates to the structure of the dislocation constriction as a function of applied shear strain.

This study also showed that absorption mechanisms A, A' and A" produce more pronounced hardening by CTBs than transmission mechanism $\mathrm{T}$; however, differences between the three absorption mechanisms are negligible. Therefore, this study infers that the primary contribution of $0^{\circ}$ kink defects to the hardening behavior of nanotwinned fcc metals is the efficiency of dislocation reactions Eqs. (5)-(6) in permanently changing the transmission mechanism into one of the three possible absorption mechanisms.

\subsection{Influence of $60^{\circ} \mathrm{kink} T B$ defects on interaction and hardening mechanisms}


Dislocation interactions with $60^{\circ}$ kink defects is the most relevant slip mode for nanotwinned fcc metals. The present study finds that this type of defect induces two major changes in dislocation behavior at the CTB-slip intersection. First, at high strain of $2 \%$, each kink step is effective in pinning transmitted dislocations to form extended jogged dislocations. From our study, it was not possible to distinguish whether the pinning is short-lived or not until the dislocation encounters another TB. However, Fig. 9 proves that hardening by dislocation pinning from TB defects is relatively moderate. Second, at intermediate strain of $1.2 \%$, another conclusion is that an entire segment of dislocation can be trapped by absorption between two kink steps, creating a sessile stair-rod dislocation in this process, which makes harder for the transmitted segments to glide in the twin grain. Here, unpinning the dislocation requires an additional reaction to eliminate the stair-rod in the absorbed segment. Fig.

9 confirms that this situation produces maximum hardening effects for $60^{\circ}$ kink defects. Third, at low strain of $0.8 \%$, all processes involve dislocation absorption, while adding TB defects did not change the mechanical behavior significantly. Similar to $0^{\circ}$ kink defects, the $60^{\circ}$ kink defects have proven to play a more significant role in strain hardening of nanotwinned $\mathrm{Cu}$ when the applied stress or strain is high, i.e. more likely as $\lambda$ decreases to the nanoscale.

\subsection{Self-migration of kink TB defects}

Self-migration of TB steps was observed in Fig. 4(b) for $0^{\circ}$ kink defects subjected to high strain $(2 \%)$, without being intersected by the screw dislocation. Fig. 8 also predicted self-migration of $60^{\circ}$ kink defects at high strain, admitting that it was more limited than for $0^{\circ}$ kinks, because of kink pinning from the fixed atom displacements 
on the upper and lower boundaries. The propagation of kink defects during relaxation is important because this process could lead to additional ductility for nanotwinned metals, similar to ITBs migration in columnar-grained fcc metals observed at high stresses during nanoindentation [41,52] and pure tensile deformation [38]. Also, TB kink movement was observed at elevated temperature [53]. The present study, however, was conducted below $3 \mathrm{~K}$, so the role of temperature is considered negligible. It has also been proposed in the literature that ITB migration requires to move the $b_{1}, b_{2}$ and $b_{3}$ partials under mechanical contributions from the applied shear strain, dislocation-dislocation interactions, Peierls-type friction forces, and interface tension of the stacking fault formed when the partial dislocation glides away from the ITB [41]. Here, our study suggests that self-migration of kink-like TB steps directly relates to mechanical shearing effects acting with respect to the direction of the step line. By considering the change from global $(x y z)$ to local $\left(x^{\prime} y^{\prime} z^{\prime}\right)$ coordinate systems, as shown in Fig. 2(b), it is possible to demonstrate that a shear strain $\varepsilon$ applied to the model produces local shear strains on the kink step such as

$$
\left[\begin{array}{ccc}
0 & \frac{2 \sqrt{2}}{3} \varepsilon & 0 \\
\frac{2 \sqrt{2}}{3} \varepsilon & 0 & \frac{1}{3} \varepsilon \\
0 & \frac{1}{3} \varepsilon & 0
\end{array}\right]_{x^{\prime} y^{\prime} z^{\prime}}
$$

Therefore, the maximum resolved shear strain acting on the step is $\varepsilon_{\mathrm{x}^{\prime} \mathrm{y}^{\prime}} \approx 0.94 \varepsilon$. To better understand the role of shear loading on the step deformation, we have also simulated the plastic deformation of a $\mathrm{Cu}$ bicrystal containing a single $\mathrm{CTB}$ with one kink step subjected to pure shear loading in the $x^{\prime} y^{\prime}$ plane, and found that this 
shearing mode induces self-migration (Movie S5). By contrast, however, applying pure shear loading in a different plane, i.e. the $y^{\prime} z$ ' plane, produced splitting of the $b_{3}: b_{1}: b_{2}$ dislocations without self-migration (Movie S6). As such, we attribute the migration of kink steps along CTBs in nanotwinned metals to mechanical shearing effects that can best accommodate the strain relaxation [53]. Therefore, an important implication of this study is that, as $\lambda$ becomes small, more self-migration of kink steps could spontaneously occur at high strains in nanotwinned $\mathrm{Cu}$ by shearing, which is consistent with the simultaneous rise in strength and ductility observed in past experiments when $\lambda$ decreased to the nanoscale $[9,11]$.

\section{Conclusion}

MD simulations in nanotwinned $\mathrm{Cu}$ bicrystals have shown that $0^{\circ}$ and $60^{\circ}$ kink $\mathrm{TB}$ defects are effective in fundamentally changing the interaction mechanism between a screw dislocation and a CTB from direct transmission to absorption, and predicted new absorption mechanisms involving the product of three twinning dislocations, instead of two for perfect CTBs. A major conclusion is that the role of kink TB defects in strengthening of nanotwinned $\mathrm{Cu}$ strongly depends on the applied strain. Kink TB defects may only successfully harden nanotwinned $\mathrm{Cu}$ at intermediate and high applied strain where direct dislocation transmission is expected with perfect CTBs, while they neither produce hardening nor softening at low applied strain. These findings suggest that the effects of kink TB defects on deformation mechanisms of nanotwinned $\mathrm{Cu}$ should become more pronounced as the twin boundary spacing decreases and strain hardening increases, or when the TB defect density increases during processing. Furthermore, self-migration of kink steps 
attributed to mechanical shearing effects was found manifest at high strain. This study has proven that new interaction mechanisms emerging from kink TB defects could play significant roles on hardening behavior and twin stability in nanotwinned $\mathrm{Cu}$, which are critically important for understanding size-dependent strength and ductility in nanotwinned fcc metals.

\section{Acknowledgments}

Support from NSF grant DMR-1410646 and the computational resources provided by Extreme Science and Engineering Discovery Environment (XSEDE) supported by NSF grant number ACI-1053575 are gratefully acknowledged. 


\section{Figure Captions}

Fig. 1. Description of slip systems at the intersection of a dislocation and a coherent twin boundary in face-centered cubic metals. (a) Double Thompson tetrahedron illustrating different $\{111\}$ slip planes on both sides of a coherent twin boundary (ABC). (b) Two-dimensional representation of one of the Thompson tetrahedrons in (a) showing relevant crystal orientations and Burgers vectors. (c) Model of a $\mathrm{Cu}$ bicrystal containing two coherent twin boundaries (TBs) used in this study for molecular dynamics simulation. (d) The atomistic model was deformed with a rigid constant shear strain by applying a linear displacement $(\delta)$ in the $y$ direction.

Fig. 2. MD simulation set-up for defective CTBs. (a) Schematic and (b) corresponding MD model of a $0^{\circ}$ kinked CTB with kink step at distance $x$ from the slip plane of a screw dislocation. Atoms with dark-blue, light-blue and red colors represent atoms in fcc, hcp and disordered arrangements, respectively. (c) Atomistic details of a kink step made of partial dislocations $b_{2}: b_{1}: b_{3}$ on each (111) plane. Atoms in the matrix and twin grains are shown with different solid and empty symbols, respectively. Coherent and incoherent TB segments are delineated by solid lines. (d) Schematic and (e) corresponding MD model of a $60^{\circ}$ kinked CTB. Two kink-like defects were introduced in such a way to maintain periodic boundary conditions in the $y$ direction. Fcc atoms in (e) have been removed for clarity. Arrows 1 and 2 represent two viewing angles for atomistic snapshots in the following figures. 
Fig. 3. Intersection of a screw dislocation with perfect CTBs. (a) $0.8 \%$ applied shear strain. (b) 1.2\% applied shear strain. CNA coloring is used in left columns 1-3, while the right column represents the atomic von-Mises shear strain.

Fig. 4. Intersection of a screw dislocation with a $0^{\circ}$ kinked CTB at $x=37 \AA$. (a) $0.8 \%$ applied shear strain. (b) $1.2 \%$ applied shear strain. CNA coloring is used in left columns 1-3, while the right column represents the atomic von-Mises shear strain.

Fig. 5. Intersection of a screw dislocation with a $0^{\circ}$ kinked CTB at $x=0$. (a) $0.8 \%$, (b) $1.2 \%$ and (c) $2.0 \%$ applied shear strain. CNA coloring is used in left columns 1-3, while the right column represents the atomic von-Mises shear strain.

Fig. 6. Intersection of a screw dislocation with a $60^{\circ}$ kinked CTB under shear strain of $\mathbf{0 . 8 \%}$. Snapshots for columns 1 and 2 are taken along views 1 and 2 as shown in Fig. 2(e), respectively. The right column is derived from DXA where dislocations are colored in accordance with colors of slip planes in Fig. 1(c).

Fig. 7. Intersection of a screw dislocation with a $60^{\circ}$ kinked CTB under shear strain of $\mathbf{1 . 2 \%}$. Snapshots for columns 1 and 2 are taken along views 1 and 2 as shown in Fig. 2(e), respectively. The right column is derived from DXA where dislocations are colored in accordance with colors of slip planes in Fig. 1(c).

Fig. 8. Intersection of a screw dislocation with a $60^{\circ}$ kinked CTB under shear strain of 2.0\%. Snapshots for columns 1 and 2 are taken along views 1 and 2 as shown in Fig. 2(e), respectively. The right column is derived from DXA where dislocations are colored in accordance with colors of slip planes in Fig. 1(c). 
Fig. 9. Dynamic stress relaxation under constant applied shear strain in all atomistic models. 


\section{References}

[1] E.L. Wood, F. Sansoz, Growth and properties of coherent twinning superlattice nanowires, Nanoscale. 4 (2012) 5268-5276.

[2] D.C. Bufford, Y.M. Wang, Y. Liu, L. Lu, Synthesis and microstructure of electrodeposited and sputtered nanotwinned face-centered-cubic metals, MRS Bull. 41 (2016) 286-291.

[3] F. Sansoz, H. Huang, D.H. Warner, An atomistic perspective on twinning phenomena in nano-enhanced fcc metals, JOM. 60 (2008) 79-84.

[4] Q. Yu, L. Qi, K. Chen, R.K. Mishra, J. Li, A.M. Minor, The nanostructured origin of deformation twinning, Nano Lett. 12 (2012) 887-892.

[5] Y. Zhao, T.A. Furnish, M.E. Kassner, A.M. Hodge, Thermal stability of highly nanotwinned copper: The role of grain boundaries and texture, J. Mater. Res. 27 (2012) 3049-3057.

[6] Y. Chen, K.Y. Yu, Y. Liu, S. Shao, H. Wang, M.A. Kirk, et al., Damagetolerant nanotwinned metals with nanovoids under radiation environments, Nat. Commun. 6 (2015) 7036.

[7] K.Y. Yu, D. Bufford, C. Sun, Y. Liu, H. Wang, M.A Kirk, et al., Removal of stacking-fault tetrahedra by twin boundaries in nanotwinned metals, Nat. Commun. 4 (2013) 1377.

[8] S. Aubry, C.J. Kimmer, A. Skye, P.K. Schelling, Comparison of theoretical and simulation-based predictions of grain-boundary Kapitza conductance in silicon, 
Phys. Rev. B. 78 (2008) 064112.

[9] L. Lu, Y. Shen, X. Chen, L. Qian, K. Lu, Ultrahigh Strength and High Electrical Conductivity in Copper, Science. 304 (2004) 422-426.

[10] Y. Zhao, I.C. Cheng, M.E. Kassner, A.M. Hodge, The effect of nanotwins on the corrosion behavior of copper, Acta Mater. 67 (2014) 181-188.

[11] L. Lu, X. Chen, X. Huang, K. Lu, Revealing the Maximum Strength in nanotwinned copper, Science. 323 (2009) 607-610.

[12] K.A. Afanasyev, F. Sansoz, Strengthening in Gold Nanopillars with Nanoscale Twins, Nano Lett. 7 (2007) 2056-2062.

[13] C. Deng, F. Sansoz, Enabling ultrahigh plastic flow and work hardening in twinned gold nanowires., Nano Lett. 9 (2009) 1517-1522.

[14] D. Jang, X. Li, H. Gao, J.R. Greer, Deformation mechanisms in nanotwinned metal nanopillars, Nat. Nanotechnol. 7 (2012) 594-601.

[15] Z.S. You, L. Lu, K. Lu, Tensile behavior of columnar grained $\mathrm{Cu}$ with preferentially oriented nanoscale twins, Acta Mater. 59 (2011) 6927-6937.

[16] Z. You, X. Li, L. Gui, Q. Lu, T. Zhu, H. Gao, L. Lu, Plastic anisotropy and associated deformation mechanisms in nanotwinned metals, Acta Mater. 61 (2013) 217-227.

[17] J.C. Ye, Y.M. Wang, T.W. Barbee Jr., A.V. Hamza, Orientation-dependent hardness and strain rate sensitivity in nanotwin copper, Appl. Phys. Lett. 100 (2012) 261912. 
[18] S. Zhang, J. Zhou, L. Wang, Y. Wang, The effect of the angle between loading axis and twin boundary on the mechanical behaviors of nanotwinned materials, Mater. Des. 45 (2013) 292-299.

[19] A. Misra, J.P. Hirth, R.G. Hoagland, Length-scale-dependent deformation mechanisms in incoherent metallic multilayered composites, Acta Mater. 53 (2005) 4817-4824.

[20] I. Shabib, R.E. Miller, Deformation characteristics and stress-strain response of nanotwinned copper via molecular dynamics simulation, Acta Mater. 57 (2009) 4364-4373.

[21] A. Stukowski, K. Albe, D. Farkas, Nanotwinned fcc metals: Strengthening versus softening mechanisms, Phys. Rev. B. 82 (2010) 224103.

[22] F. Sansoz, K. Lu, T. Zhu, A. Misra, Strengthening and plasticity in nanotwinned metals, MRS Bull. 41 (2016) 292-297.

[23] V. Borovikov, M.I. Mendelev, A.H. King, R. LeSar, Effects of Schmid factor and slip nucleation on deformation mechanism in columnar-grained nanotwinned Ag and Cu, J. Appl. Phys. 117 (2015) 085302.

[24] X. Li, Y. Wei, L. Lu, K. Lu, H. Gao, Dislocation nucleation governed softening and maximum strength in nano-twinned metals, Nature. 464 (2010) 877-880.

[25] N. Lu, K. Du, L. Lu, H.Q. Ye, Transition of dislocation nucleation induced by local stress concentration in nanotwinned copper, Nat. Commun. 6 (2015) 7648.

[26] H. Zhou, X. Li, S. Qu, W. Yang, H. Gao, A Jogged Dislocation Governed 
Strengthening Mechanism in Nanotwinned Metals, Nano Lett. 14 (2014) 50755800.

[27] Z. Chen, Z. Jin, H. Gao, Repulsive force between screw dislocation and coherent twin boundary in aluminum and copper, Phys. Rev. B. 75 (2007) 212104.

[28] Y.T. Zhu, X.L. Wu, X.Z. Liao, J. Narayan, L.J. Kecskés, S.N. Mathaudhu, Dislocation-twin interactions in nanocrystalline fcc metals, Acta Mater. 59 (2011) 812-821.

[29] T. Zhu, J. Li, A. Samanta, H.G. Kim, S. Suresh, Interfacial plasticity governs strain rate sensitivity and ductility in nanostructured metals., Proc. Natl. Acad. Sci. U. S. A. 104 (2007) 3031-3036.

[30] Z.H. Jin, P. Gumbsch, E. Ma, K. Albe, K. Lu, H. Hahn, H. Gleiter, The interaction mechanism of screw dislocations with coherent twin boundaries in different face-centred cubic metals, Scr. Mater. 54 (2006) 1163-1168.

[31] M. Chassagne, M. Legros, D. Rodney, Atomic-scale simulation of screw dislocation/coherent twin boundary interaction in $\mathrm{Al}, \mathrm{Au}, \mathrm{Cu}$ and $\mathrm{Ni}$, Acta Mater. 59 (2011) 1456-1463.

[32] Z.H. Jin, P. Gumbsch, K. Albe, E. Ma, K. Lu, H. Gleiter, et al., Interactions between non-screw lattice dislocations and coherent twin boundaries in facecentered cubic metals, Acta Mater. 56 (2008) 1126-1135.

[33] V. Yamakov, D. Wolf, S.R. Phillpot, H. Gleiter, Dislocation-dislocation and dislocation-twin reactions in nanocrystalline Al by molecular dynamics 
simulation, Acta Mater. 51 (2003) 4135-4147.

[34] Y.G. Zheng, J. Lu, H.W. Zhang, Z. Chen, Strengthening and toughening by interface-mediated slip transfer reaction in nanotwinned copper, Scr. Mater. 60 (2009) 508-511.

[35] Z.X. Wu, Y.W. Zhang, D.J. Srolovitz, Deformation mechanisms, length scales and optimizing the mechanical properties of nanotwinned metals, Acta Mater. 59 (2011) 6890-6900.

[36] S. Ni, Y.B. Wang, X.Z. Liao, R.B. Figueiredo, H.Q. Li, S.P. Ringer, et al., The effect of dislocation density on the interactions between dislocations and twin boundaries in nanocrystalline materials, Acta Mater. 60 (2012) 3181-3189.

[37] J. Wang, X. Zhang, Twinning effects on strength and plasticity of metallic materials, MRS Bull. 41 (2016) 274-281.

[38] Y.M. Wang, F. Sansoz, T. LaGrange, R.T. Ott, J. Marian, T.W. Barbee Jr., et al., Defective twin boundaries in nanotwinned metals, Nat. Mater. 12 (2013) $697-702$.

[39] O. Anderoglu, A. Misra, H. Wang, F. Ronning, M.F. Hundley, X. Zhang, Epitaxial nanotwinned $\mathrm{Cu}$ films with high strength and high conductivity, Appl. Phys. Lett. 93 (2008) 083108.

[40] J. Wang, O. Anderoglu, J.P. Hirth, A. Misra, X. Zhang, Dislocation structures of $\Sigma 3\{112\}$ twin boundaries in face centered cubic metals, Appl. Phys. Lett. 95 (2009) 021908.

[41] J. Wang, N. Li, O. Anderoglu, X. Zhang, A. Misra, J.Y. Huang, J.P. Hirth, 
Detwinning mechanisms for growth twins in face-centered cubic metals, Acta Mater. 58 (2010) 2262-2270.

[42] X.H. An, M. Song, Y. Huang, X.Z. Liao, S.P. Ringer, T.G. Langdon, Y.T. Zhu, Twinning via the motion of incoherent twin boundaries nucleated at grain boundaries in a nanocrystalline $\mathrm{Cu}$ alloy, Scr. Mater. 72-73 (2014) 35-38.

[43] S.J. Plimpton, Fast parallel algorithms for short-range molecular dynamics, J. Comput. Phys. 117.1 (1995) 1-19.

[44] Y. Mishin, M. Mehl, D. Papaconstantopoulos, A.F. Voter, J.D. Kress, Structural stability and lattice defects in copper: Ab initio, tight-binding, and embedded-atom calculations, Phys. Rev. B. 63 (2001) 224106.

[45] R.D. Boyer, J. Li, S. Ogata, S. Yip, Analysis of shear deformations in Al and $\mathrm{Cu}$ : empirical potentials versus density functional theory, Model. Simul. Mater. Sci. Eng. 12 (2004) 1017-1029.

[46] D. Faken, H. Jónsson, Systematic analysis of local atomic structure combined with 3D computer graphics, Comput. Mater. Sci. 2 (1994) 279-286.

[47] H. Tsuzuki, P.S. Branicio, J.P. Rino, Structural characterization of deformed crystals by analysis of common atomic neighborhood, Comput. Phys. Commun. 177 (2007) 518-523.

[48] A. Stukowski, K. Albe, Dislocation detection algorithm for atomistic simulations, Model. Simul. Mater. Sci. Eng. 18 (2010) 025016.

[49] A. Stukowski, V.V. Bulatov, A. Arsenlis, Automated identification and indexing of dislocations in crystal interfaces, Model. Simul. Mater. Sci. Eng. 
(2012) 085007.

[50] A. Stukowski, Visualization and analysis of atomistic simulation data with OVITO-the Open Visualization Tool, Model. Simul. Mater. Sci. Eng. 18 (2010) 015012.

[51] J.P. Hirth, J. Lothe, Theory of dislocations, 2nd ed., New York: Wiley; 1982.

[52] J. Wang, A. Misra, J.P. Hirth, Shear response of $\Sigma 3\{112\}$ twin boundaries in face-centered-cubic metals, Phys. Rev. B. 83 (2011) 064106.

[53] L. Xu, D. Xu, K.N. Tu, Y. Cai, N. Wang, P. Dixit, et al., Structure and migration of (112) step on (111) twin boundaries in nanocrystalline copper, J. Appl. Phys. 104 (2008) 113717. 
(a)

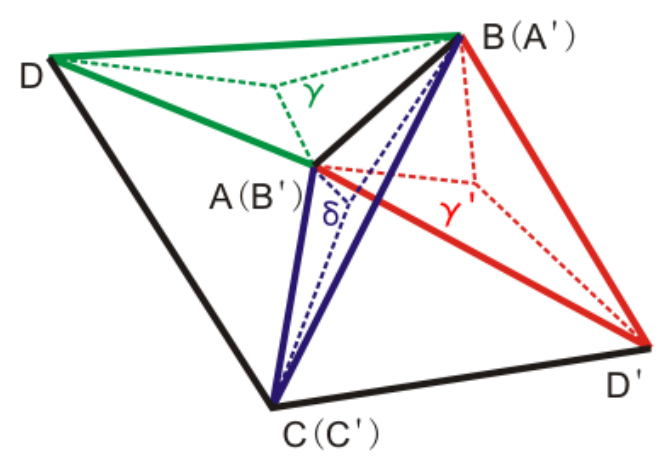

(c)

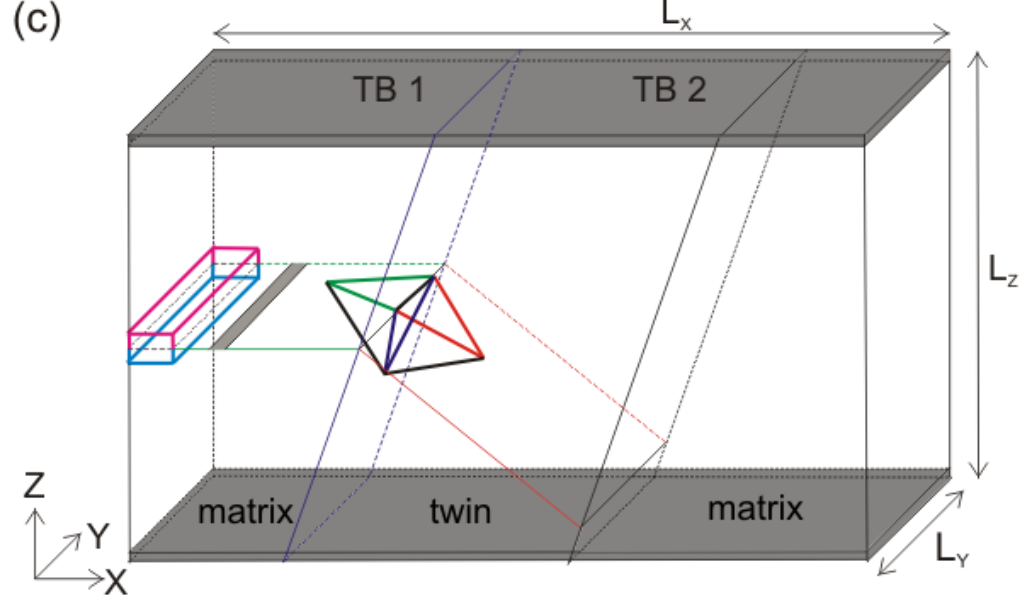

(b)

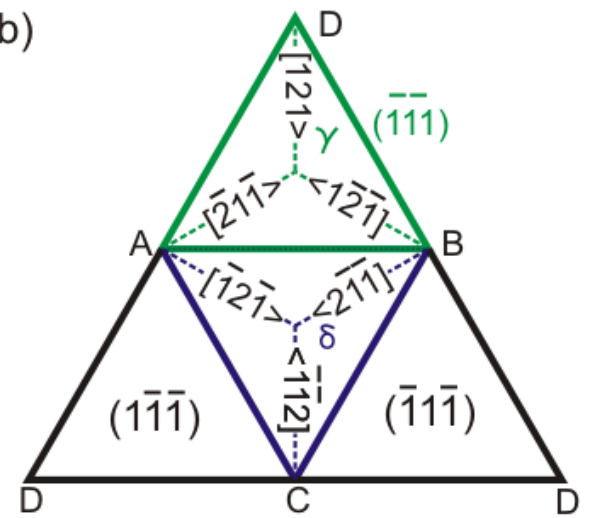

(111)

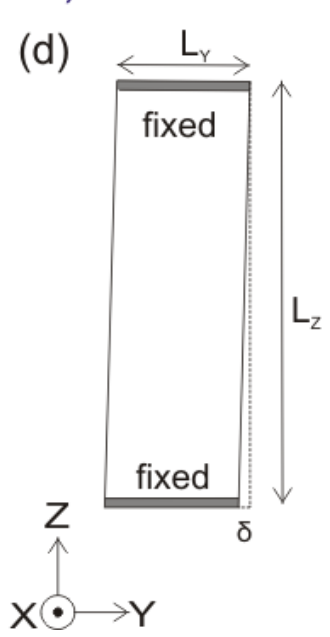

Fig. 1. Description of slip systems at the intersection of a dislocation and a coherent twin boundary in face-centered cubic metals. (a) Double Thompson tetrahedron illustrating different $\{111\}$ slip planes on both sides of a coherent twin boundary (ABC). (b) Twodimensional representation of one of the Thompson tetrahedrons in (a) showing relevant crystal orientations and Burgers vectors. (c) Model of a $\mathrm{Cu}$ bicrystal containing two coherent twin boundaries (TBs) used in this study for molecular dynamics simulation. (d) The atomistic model was deformed with a rigid constant shear strain by applying a linear displacement $(\delta)$ in the $y$ direction. 


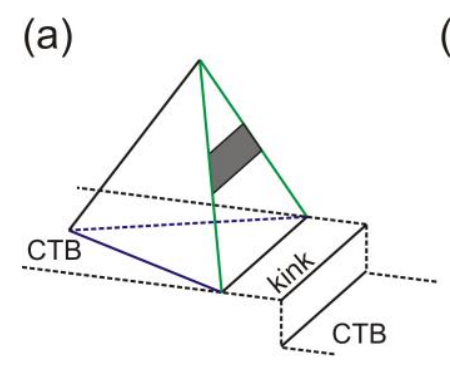

(b)

(d)

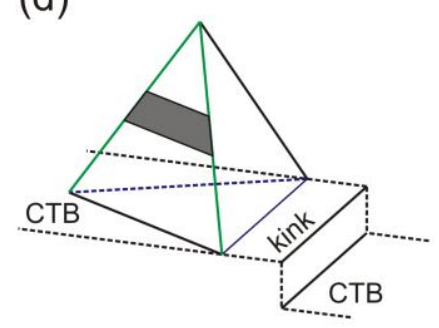

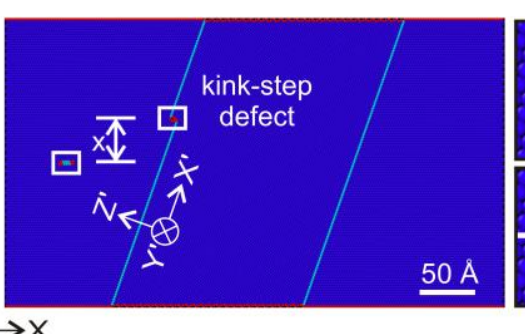
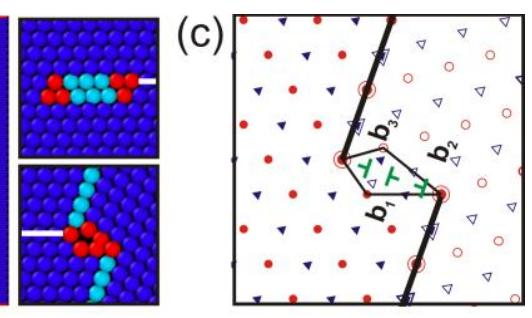

(e)

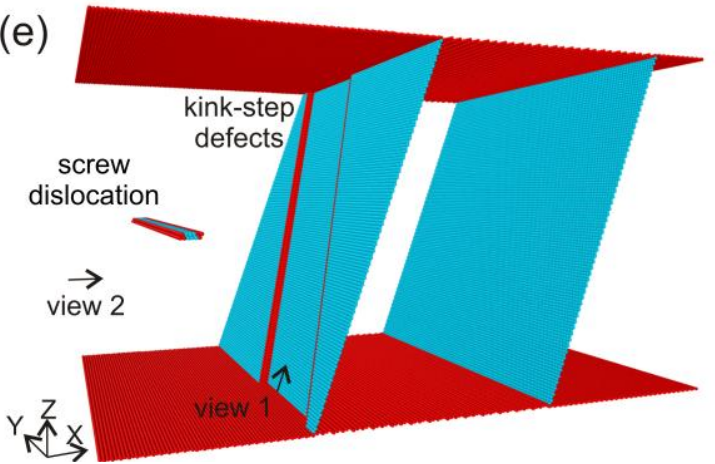

Fig. 2. MD simulation set-up for defective CTBs. (a) Schematic and (b) corresponding MD model of a $0^{\circ}$ kinked CTB with kink step at distance $x$ from the slip plane of a screw dislocation. Atoms with dark-blue, light-blue and red colors represent atoms in fcc, hep and disordered arrangements, respectively. (c) Atomistic details of a kink step made of partial dislocations $b_{2}: b_{1}: b_{3}$ on each (111) plane. Atoms in the matrix and twin grains are shown with different solid and empty symbols, respectively. Coherent and incoherent TB segments are delineated by solid lines. (d) Schematic and (e) corresponding MD model of a $60^{\circ}$ kinked CTB. Two kink-like defects were introduced in such a way to maintain periodic boundary conditions in the $y$ direction. Fcc atoms in (e) have been removed for clarity. Arrows 1 and 2 represent two viewing angles for atomistic snapshots in the following figures. 
(a)

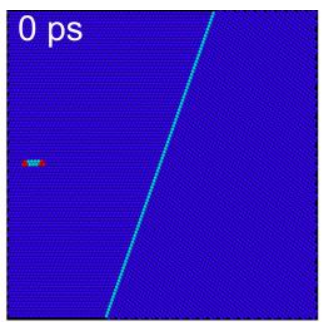

(b)

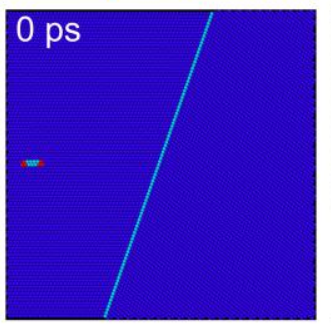

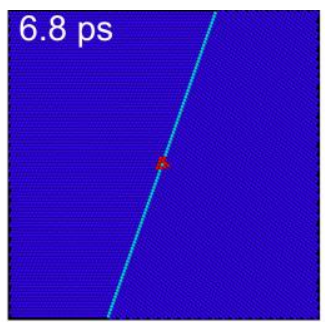

6 ps

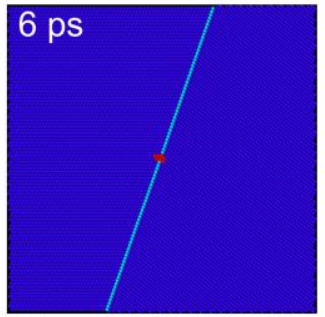

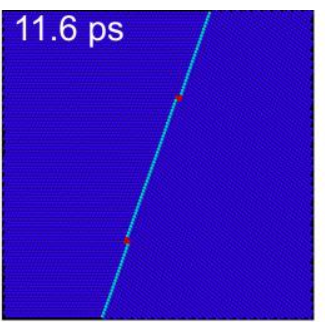

$11.6 \mathrm{ps}$

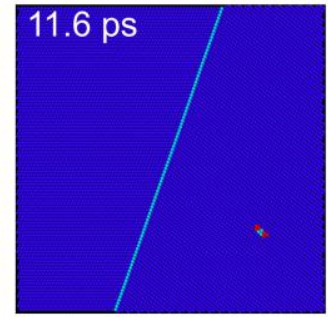

\section{$11.6 \mathrm{ps}$}

A

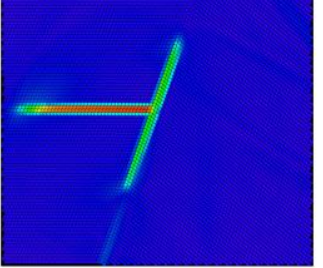

$11.6 \mathrm{ps}$

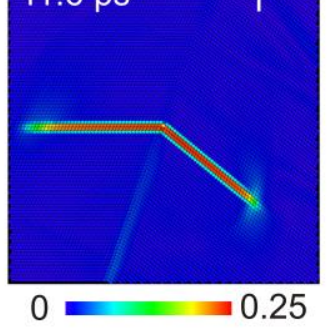

Fig. 3. Intersection of a screw dislocation with perfect CTBs. (a) $0.8 \%$ applied shear strain. (b) $1.2 \%$ applied shear strain. CNA coloring is used in left columns $1-3$, while the right column represents the atomic von-Mises shear strain. 
(a)

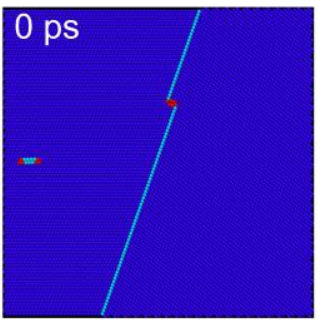

(b)

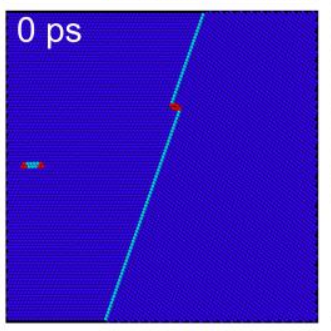

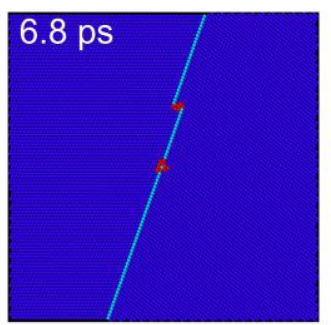

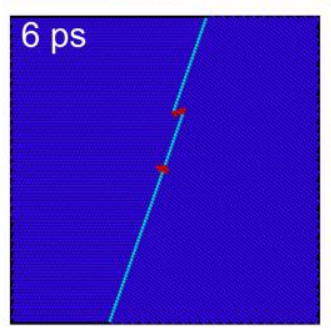

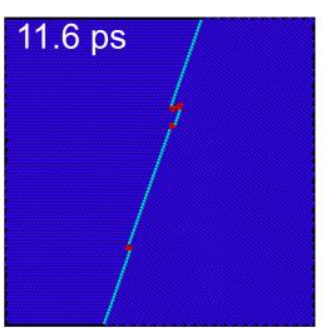

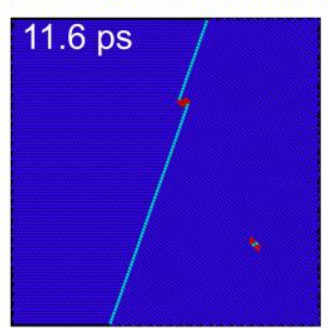

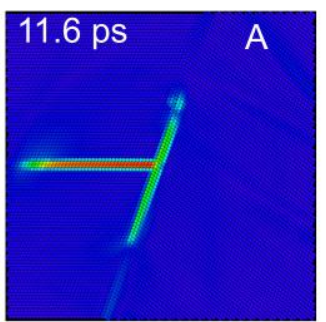

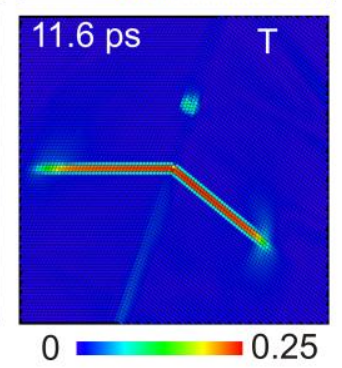

Fig. 4. Intersection of a screw dislocation with a $0^{\circ}$ kinked CTB at $x=37 \AA$. (a) $0.8 \%$ applied shear strain. (b) $1.2 \%$ applied shear strain. CNA coloring is used in left columns 1-3, while the right column represents the atomic von-Mises shear strain. 
(a)

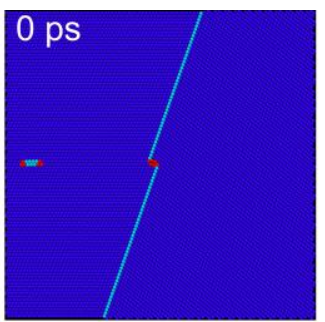

(b)

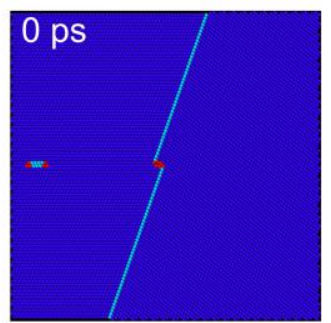

(c)

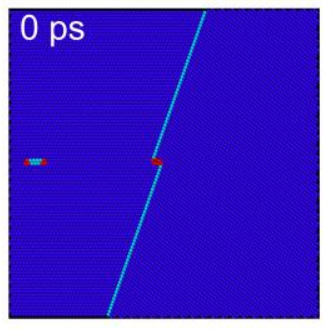

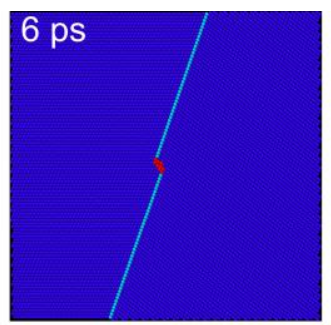
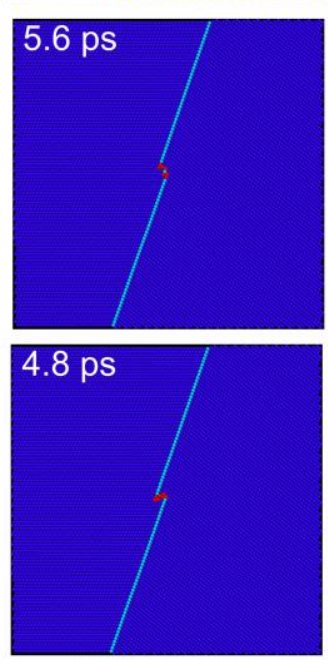
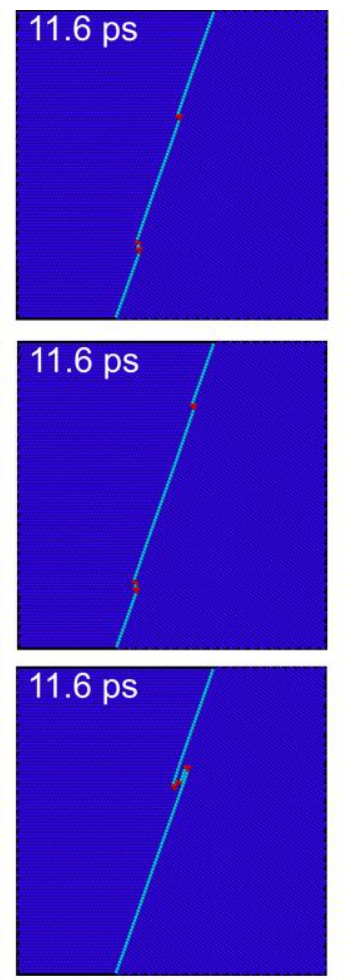

\section{$11.6 \mathrm{ps}$}
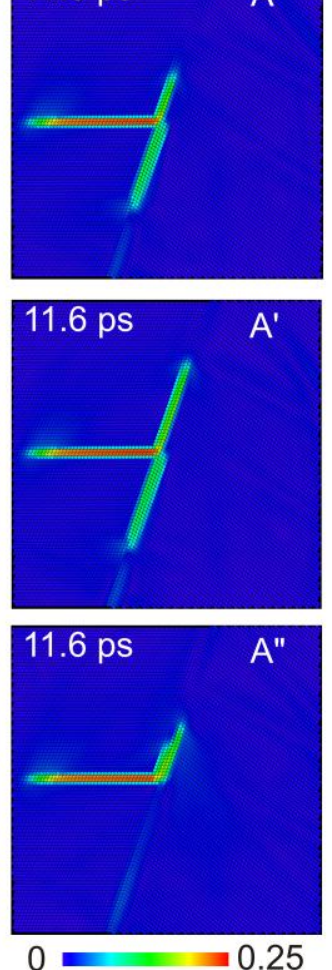

Fig. 5. Intersection of a screw dislocation with a $0^{\circ}$ kinked CTB at $x=0$. (a) $0.8 \%$, (b) $1.2 \%$ and (c) $2.0 \%$ applied shear strain. CNA coloring is used in left columns 1-3, while the right column represents the atomic von-Mises shear strain. 
(a) $0 \mathrm{ps}$
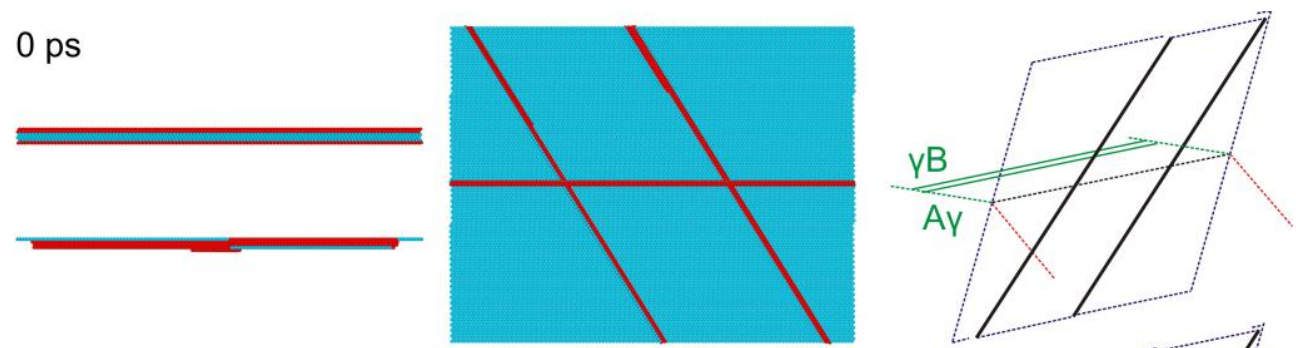

(b) $\quad 6.8 \mathrm{ps}$

A
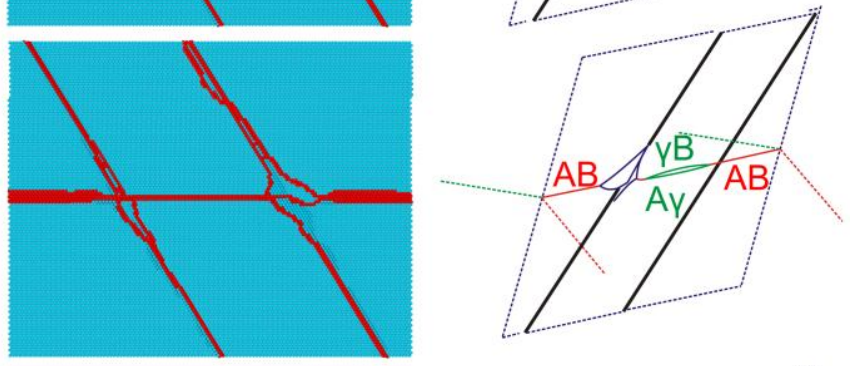

(d) $\quad 11.6 \mathrm{ps}$

A
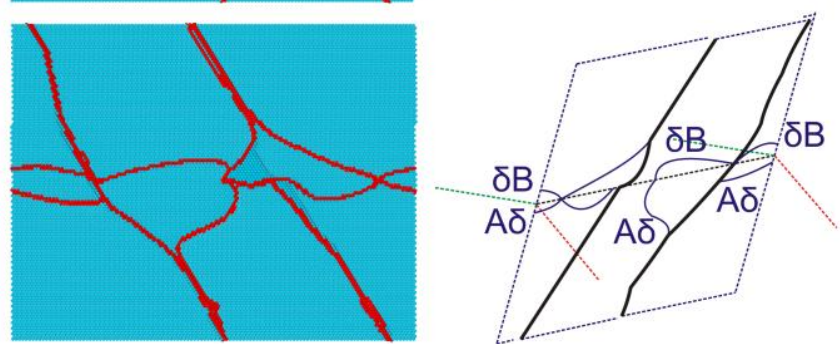

Fig. 6. Intersection of a screw dislocation with a $60^{\circ}$ kinked CTB under shear strain of $\mathbf{0 . 8 \%}$. Snapshots for columns 1 and 2 are taken along views 1 and 2 as shown in Fig. 2(e), respectively. The right column is derived from DXA analysis where dislocations are colored in accordance with colors of slip planes in Fig. 1(c). 
(a) $0 \mathrm{ps}$
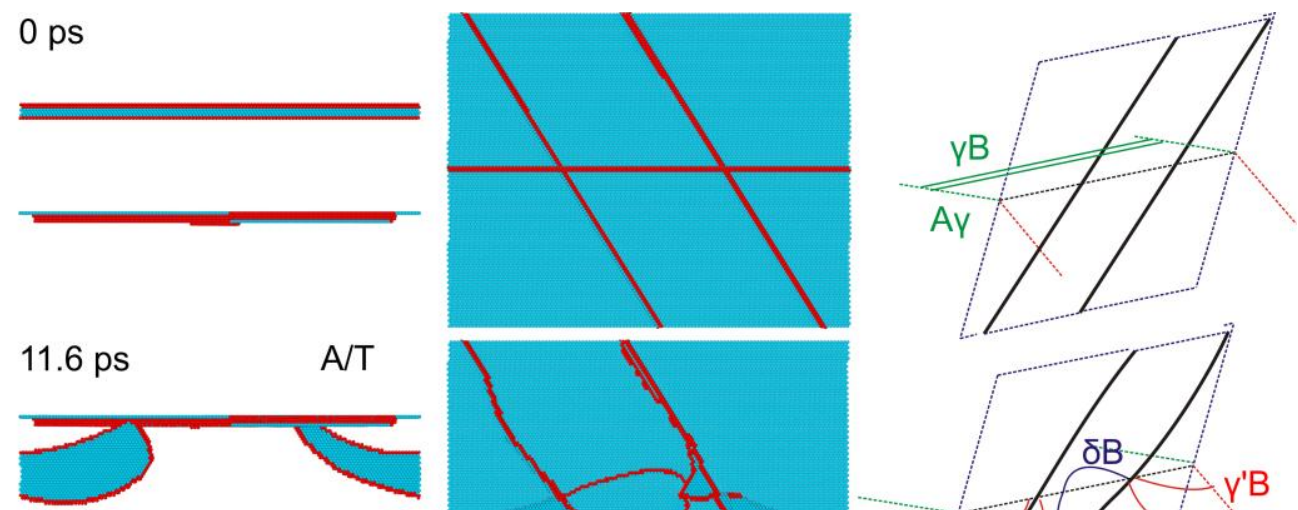

(b)
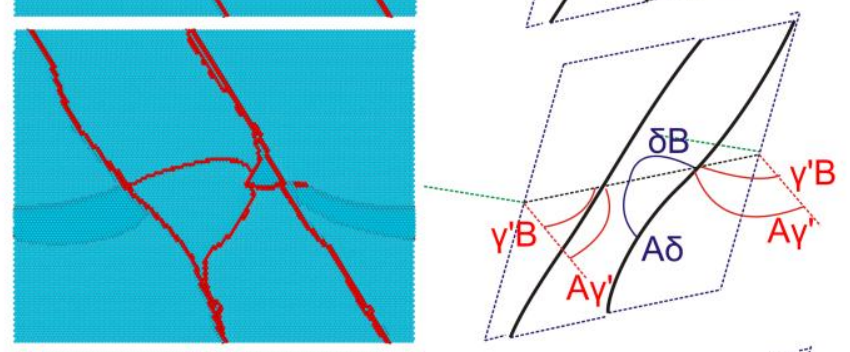

(c) $\quad 17.2 \mathrm{ps}$
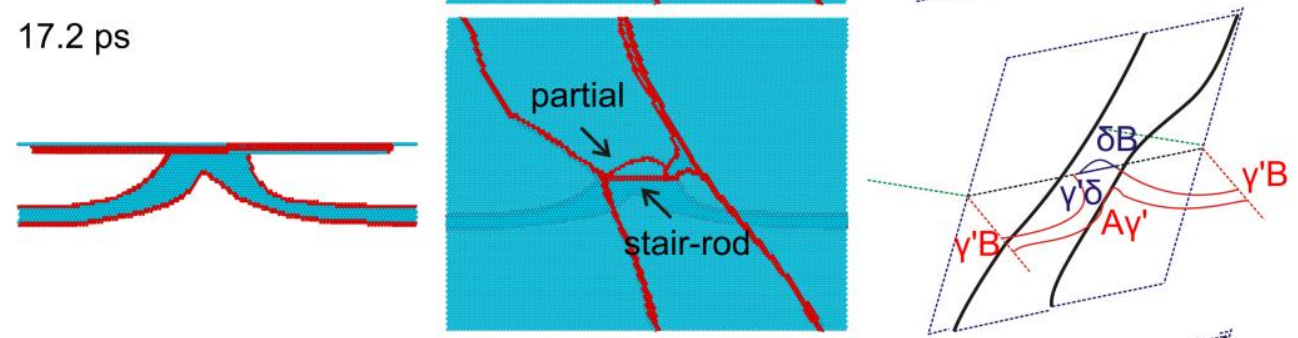

(d) $\quad 19.6 \mathrm{ps}$

T
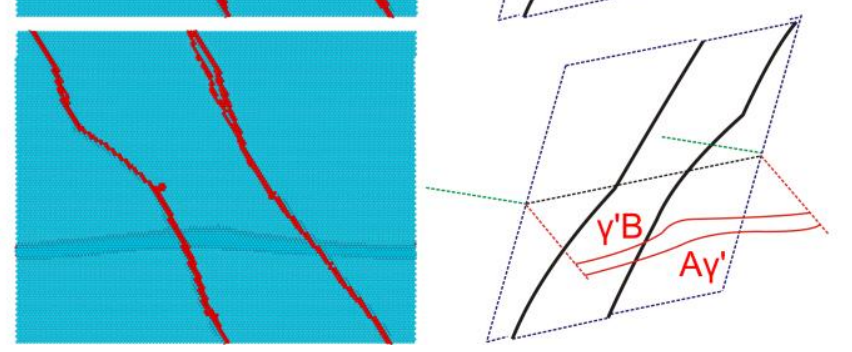

Fig. 7. Intersection of a screw dislocation with a $60^{\circ}$ kinked CTB under shear strain of 1.2\%. Snapshots for columns 1 and 2 are taken along views 1 and 2 as shown in Fig. 2(e), respectively. The right column is derived from DXA analysis where dislocations are colored in accordance with colors of slip planes in Fig. 1(c). 
(a) $0 \mathrm{ps}$
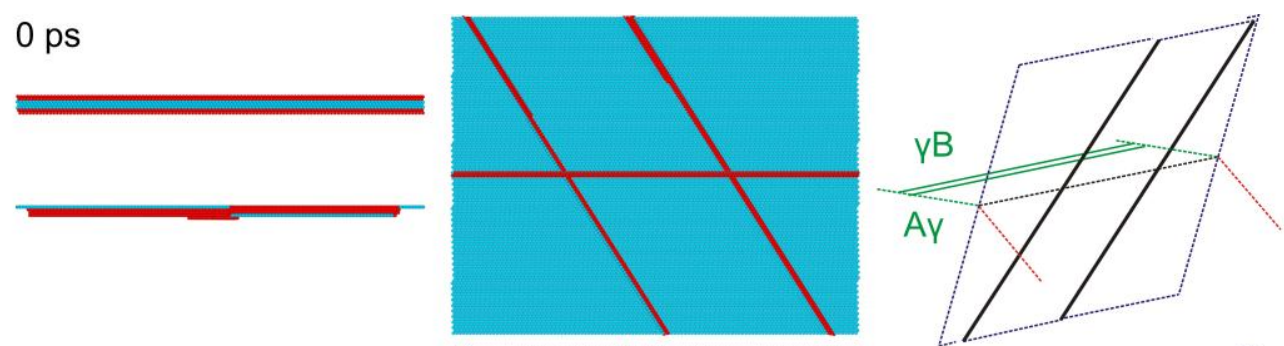

(b) $7.6 \mathrm{ps}$
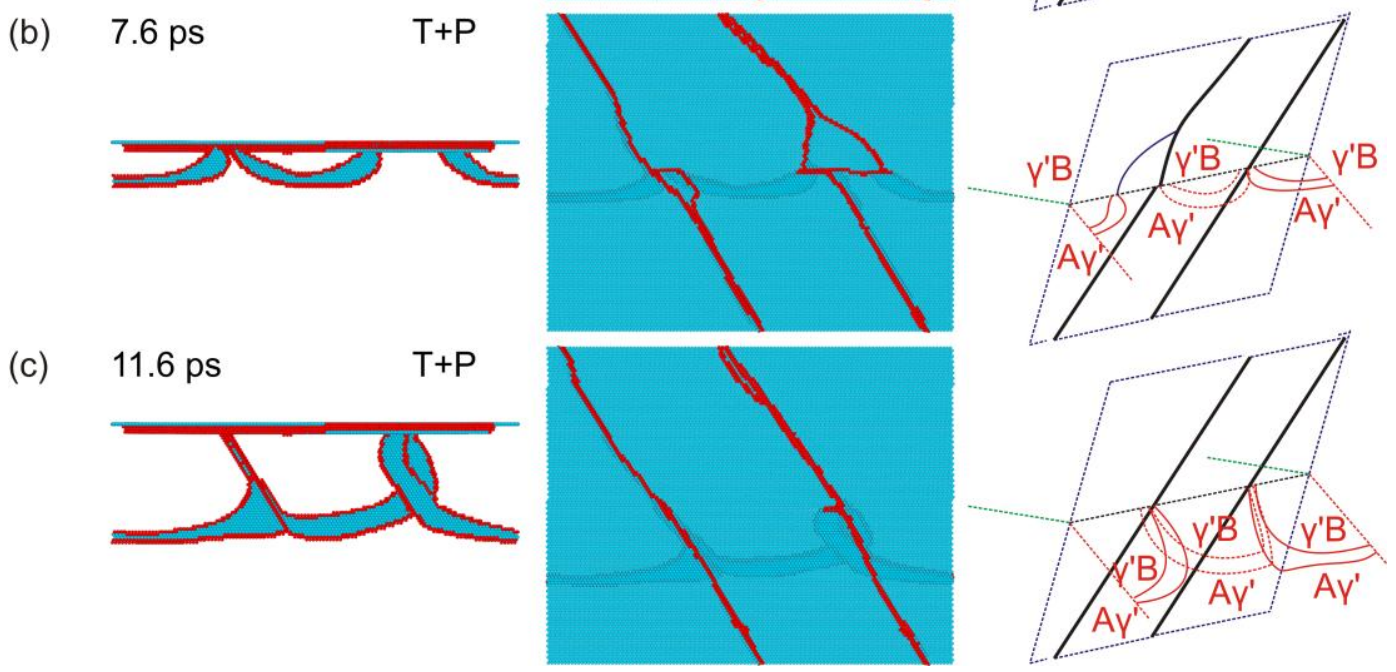

Fig. 8. Intersection of a screw dislocation with a $60^{\circ}$ kinked CTB under shear strain of 2.0\%. Snapshots for columns 1 and 2 are taken along views 1 and 2 as shown in Fig. 2(e), respectively. The right column is derived from DXA analysis where dislocations are colored in accordance with colors of slip planes in Fig. 1(c). 


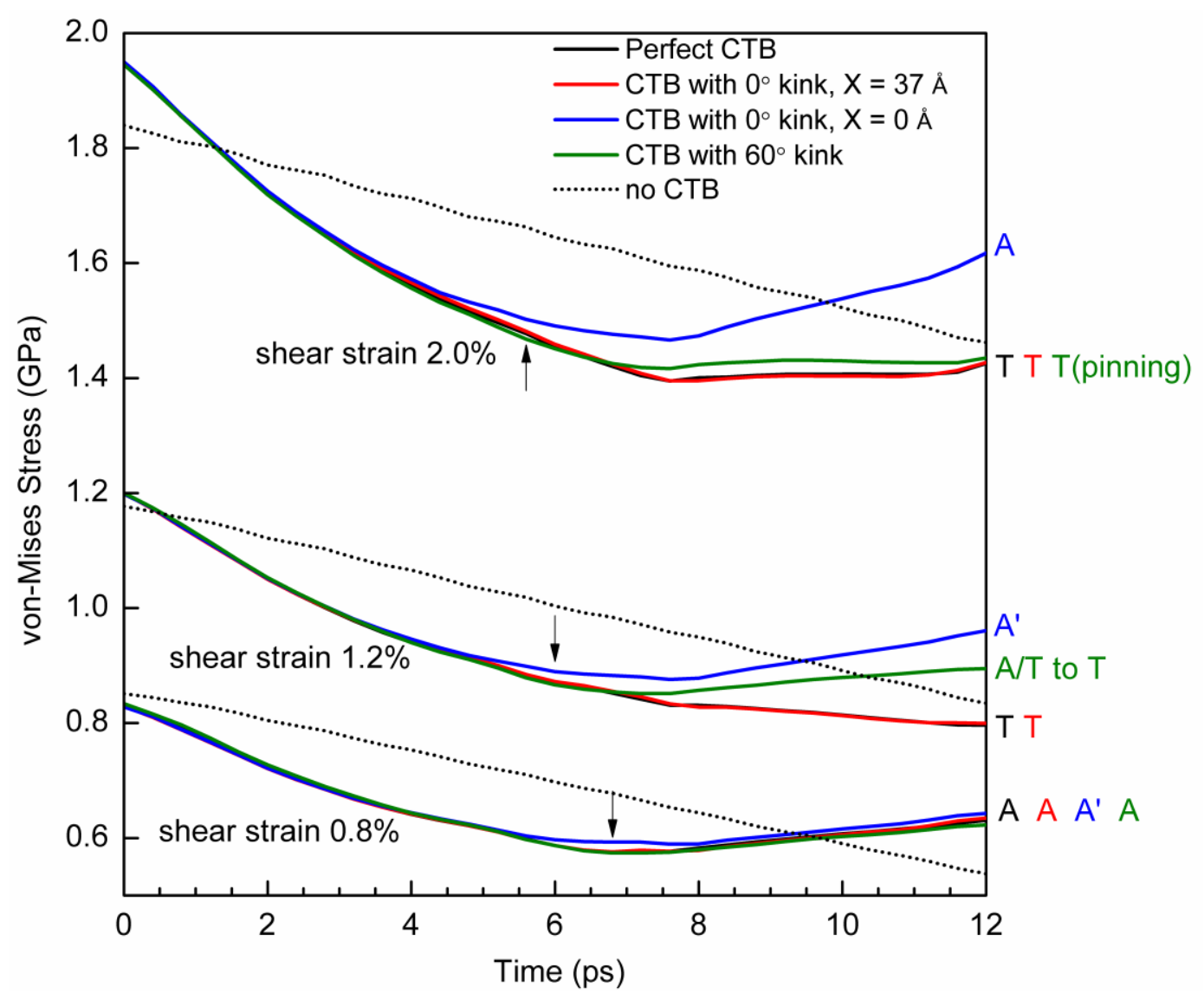

Fig. 9. Dynamic stress relaxation under constant applied shear strain in all atomistic models. 
shear strain $2.0 \%$

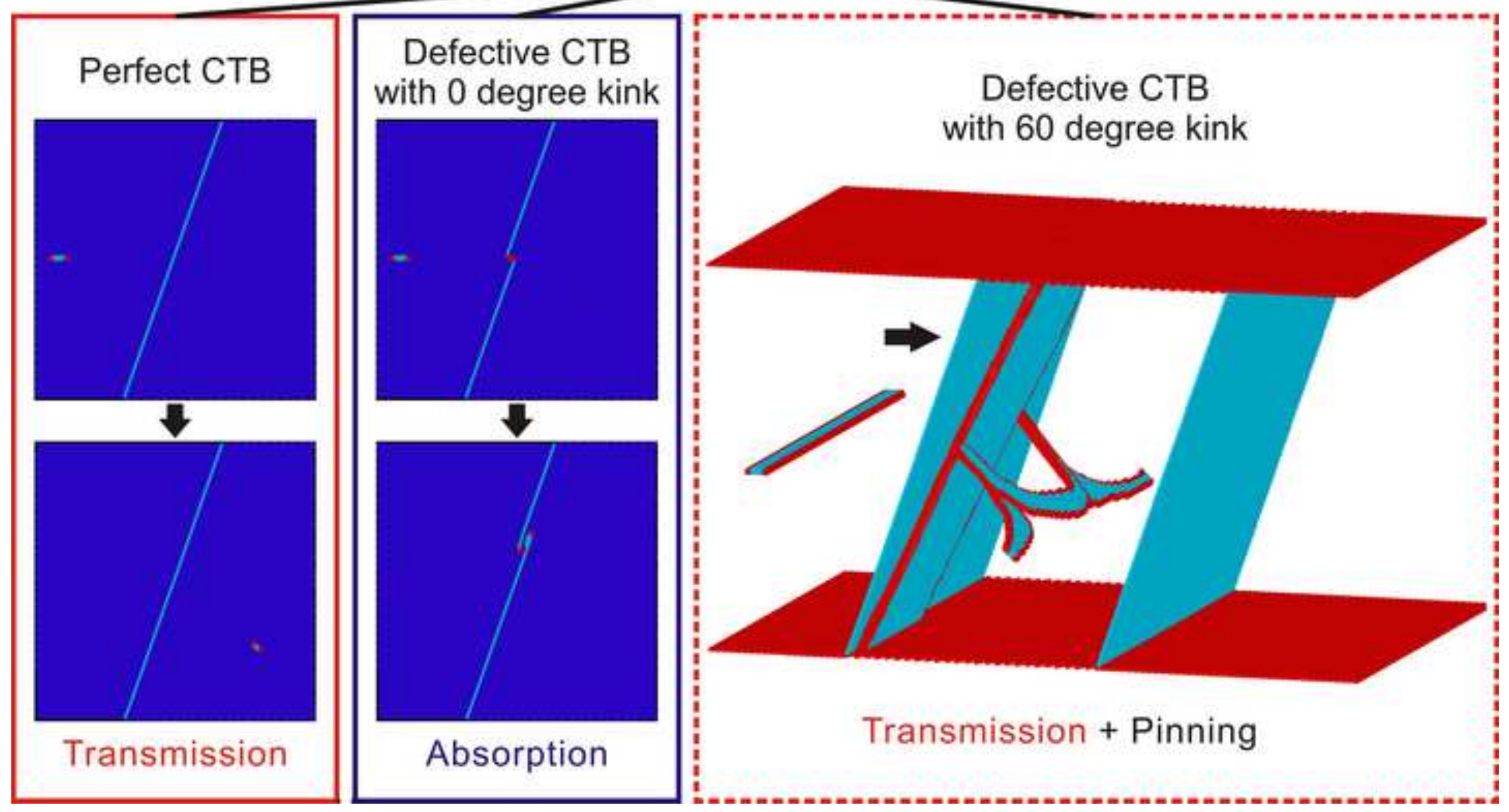

